\title{
The Optimal Subchannel and Bit Allocation Problem for OFDM
}

\author{
Taehyung Park ${ }^{1, \star}$ and Sungbin $\mathrm{Im}^{2}$ \\ ${ }^{1}$ Department of Industrial and Information Systems Engineering, \\ Soongsil University, Seoul, Korea \\ tpark@ssu.ac.kr \\ ${ }^{2}$ School of Electrical Engineering, Soongsil University, Seoul, Korea \\ sbi@nuri.net
}

\begin{abstract}
The advantages of the orthogonal frequency division multiplexing (OFDM) are high spectral efficiency, resiliency to RF interference, and lower multi-path distortion. To further utilize vast channel capacity of the multiuser OFDM, one has to find the efficient adaptive subchannel and bit allocation among users. In this paper, we propose an 0-1 integer programming model formulating the optimal subchannel and bit allocation problem of the multiuser OFDM. We provide an efficient Lagrangian dual formulation with fast solution approach. Simulation results are provided to show the effectiveness of the 0-1 integer programming model. MATLAB simulation on a system employing Mary quardarature amplitude modulation (MQAM) assuming a frequencyselective channel consisting of five independent Rayleigh multipaths are carried with the optimal subchannel and bit allocation solution generated by $0-1$ integer programming model.
\end{abstract}

\section{Introduction}

In this paper, we consider the optimal subchannel and bit allocation problem in the multiuser OFDM (Orthogonal Frequency Division Multiplexing) network. Multicarrier modulation technique is utilized for reducing intersymbol interference. Assuming that the transmitter knows the instantaneous channel transfer functions of all users, significant performance improvement is achieved if adaptive modulation is used with OFDM. Specially, subchannels with large channel gains carry more bits/OFDM symbol, while subchannels with deep fade carry one or zero bits/symbol.

Previous research on adaptive subchannel assignment and bit allocation problem are references [1, [3, 4, [5, and [7. These research can be divided into two groups; In [1], 3], and 7], the problem is defined as minimizing the total channel power with the constrains of satisfying all user's data transmission demand and exclusive channel assignment for each user; In [4] and [5], the problem is maximizing the minimum of user's throughput subject to the power constraints. In

^ Both authors are supported by the research fund from Soongsil University, Seoul, Korea. 
[1] and [7, the optimization problem is mainly formulated as a convex nonlinear programming while our model explicitly incorporates the integrality of the bit loading and channel assignment.

The remainder of this paper is organized as follows. In the next section, we introduce the assumptions underlying our model and formulate the adaptive subchannel assignment and bit loading problem into an equivalent 0-1 integer linear programming problem. In Section 3, we compare our solution with a static assignment approaches via Monte Carlo simulation.

\section{Problem Formulation}

We assume that there are $m$ users and the $i$ th user has a data rate of $R_{i}$ bits per OFDM symbol. In the transmitter, the serial data from the $m$ users are fed into subchannel and bit allocation blocks which allocate bits from different users to different subchannels. We assume that there are $n$ subchannels. The instantaneous channel information on all the subchannels of all the users is assumed to be available at the transmitter. It is also assumed that the subchannel and bit allocation information is sent to each user by a separate channel. The complex symbols at the output of the modulators are transformed into the time domain samples by inverse fast Fourier transform. Cyclic extension of the time domain samples, the guard interval, is then added to ensure orthogonality among the subchannels. The transmit signal is then passed through different frequency selective fading channels to different users.

To formulate the problem into an optimization problem, we introduce the decision variables $x_{i j}$ and $y_{i j} . x_{i j}$ denotes the number of bits/symbol of the $i$ th user that are assigned to the $j$ th subchannel and has values in $C=\{0,1, \ldots, M\}$. 0 - 1 variable $y_{i j}$ is one if user $i$ is assigned to subchannel $j$ and zero, otherwise. Since each user's data transmission requirement must be satisfied, we have $\sum_{j} x_{i j}=R_{i} \forall i$. Also, each subchannel cannot be shared by more than one user, we have $\sum_{i} y_{i j} \leq 1 \forall j$. Since the allocated number of bits $x_{i j}=0$ if user $i$ is not assigned to subchannel $j$, and if $y_{i j}=1, x_{i j}$ can be positive, we have $x_{i j} \leq M y_{i j} \forall i, j$.

The transmission power allocated to user $i$ 's subchannel $j$ is assumed to be a convex nonlinear function $f_{i}\left(x_{i j}\right) / \alpha_{i j}^{2}$ where $f_{i}\left(x_{i j}\right)$ denotes the required power supporting $x_{i j}$ bits/symbol at a given bit per error(BER) when the channel gain is equal to unity, and $\alpha_{i j}$ the instantaneous channel gain that is assumed to be known to the transmitter. The specific form of $f_{i}\left(x_{i j}\right)$ used is found on page 1752 in [7].

Instead of using a general integer variable $x_{i j}$, we can represent $x_{i j}$ in terms of 0 - 1 varibles $x_{i j k}$ with constraints $x_{i j}=\sum_{k \in C} k x_{i j k} \forall i, j, \sum_{k} x_{i j k}=1 \forall i, j$, and $x_{i j k}$ integer $\forall i, j, k$. Since $f_{i}\left(x_{i j}\right) \in\left\{0, f_{i}(1), \ldots, f_{i}(M)\right\}$, the transmission power term $f_{i}\left(x_{i j}\right) / \alpha_{i j}^{2}$ has value $c_{i j k}=f_{i}(k) / \alpha_{i, j}^{2} \forall i, j$ and $k \in\{0,1,2, \ldots, M\}$. Now the optimization problem becomes the following 0-1 integer linear programming problem. 


$$
\begin{gathered}
\sum_{i} \sum_{j} \sum_{k} c_{i j k} x_{i j k} \\
\sum_{j} \sum_{k} k x_{i j k}=R_{i} \quad \forall i \\
\sum_{k} x_{i j k} \leq y_{i j} \quad \forall i, j \\
\sum_{i} y_{i j} \leq 1 \quad \forall j \\
x_{i j k} \geq 0 \quad \forall i, j, k \\
y_{i j} \geq 0 \quad \forall i, j \\
x_{i j k} \text { integer } \quad \forall i, j, k \\
y_{i j} \text { integer } \quad \forall i, j .
\end{gathered}
$$

Note that if $m=1$, problem (11) becomes a Generalized Upper Bounded (GUB) constrained knapsack problem and 2] contains a specialized algorithm for the linear programming relaxation of formulation (1) when $m=1$.

If we discard the integrality constraints upon $x_{i j k}$ and $y_{i j}$ from the formulation (11) and dualize the constraints (1b) employing dual variables $\mu=\left(\mu_{i}\right)$, the Lagrangian subproblem has

$$
\sum_{i} \sum_{j} \sum_{k}\left(c_{i j k}+k \mu_{i}\right) x_{i j k}-\sum_{i} \mu_{i} R_{i}
$$

as the objective function and has constraints (1C) - (1f). Since the Lagrangian subproblem is separable with respect to $j$, we have the following Lagrangian dual problem LD.

$$
\text { LD: } \operatorname{Maximize}_{\mu} \quad \theta(\mu)=\sum_{j} \theta^{j}(\mu)-\sum_{i} \mu_{i} R_{i}
$$

where $\theta^{j}(\mu) \equiv \operatorname{Minimum}\left\{\sum_{i} \sum_{k}\left(c_{i j k}+k \mu_{i}\right) x_{i j k} \mid \sum_{k} x_{i j k} \leq y_{i j} \forall i ; \sum_{i} y_{i j} \leq\right.$ $\left.1 ; x_{i j k} \geq 0 \forall i, k ; y_{i j} \geq 0 \forall i\right\}$.

It can be easily proved that $\theta^{j}(\mu)=\min \left\{\min _{i, k}\left\{c_{i j k}+k \mu_{i}\right\}, 0\right\}$, so we have $\theta(\mu)=\sum_{j} \min \left\{\min _{i, k}\left\{c_{i j k}+k \mu_{i}\right\}, 0\right\}-\sum_{i} \mu_{i} R_{i}$. The Lagrangian dual LD is nondifferentiable concave maximization problem and the subgradient maximization approach such as the Variable Target Value Method(VTVM) [6] can be applied.

\section{Simulation Result and Summary}

In this section, we present simulation results to show the performance of the proposed algorithm. In the simulations presented here, the wireless channel is modeled as a frequency-selective channel consisting of five independent Rayleigh multipaths with an exponential power delay profile, which are implemented using the MATLAB functions. We consider a system that employs M-ary quadrature amplitude modulation (MQAM) with $C=\{0,2,4,6\}$. That is, 4-QAM, 16-QAM, and 64-QAM are used for carrying 2,4 , and 6 bits/symbol.

In order to evaluate the performance of the proposed algorithm, we carried out 500 independent experiments for each $E_{b} / N_{0}$. In the experiment, 128 subcarriers are used along with a total transmission rate of 512 bits/symbol over 


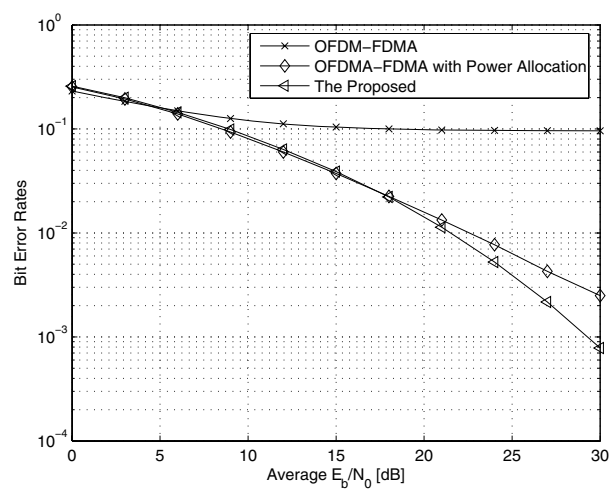

Fig. 1. Bit error rates versus $E_{b} / N_{0}$ for the three schemes

all users. The results are depicted in Fig. 1. For the purpose of comparison, the BER curves of two different schemes are provided in Fig. 11 One is "OFDMFDMA" (frequency division multiple access), which distributes the same number of bits to each subcarrier, (4 bits per subcarrier in this experiment), while "OFDM-FDMA with Power Allocation" is to employ the adaptive power allocation algorithm with OFDM-FDMA. As shown in Fig. 1, the curve of OFDMFDMA shows the error floor effect due to the frequency-selective channel. The proposed algorithm is superior to the other two methods, especially, for high $E_{b} / N_{0}$.

\section{References}

1. Aggarwal, A., Meng, T.H.: A convex interior-point method for optimal OFDM PAR reduction. IEEE International Conference on Communications (ICC) (2005)

2. Johnson E.L., Padberg M.W.: A note on the knapsack problem with special ordered sets. Oper. Res. Lett. 1 (1981) 18-22

3. Kim, I., Lee, H.L., Kim, B., Lee, Y.H.: On the use of linear programming for dynamic subchannel and bit allocation in multiuser OFDM. GLOBECOM '01, 6 (2001) 36483652

4. Rhee, W., Cioffi, J.M.: Increasing in capacity of multiuser OFDM system using dynamic subchannel allocation. Proceedings of the IEEE VTC (2000)

5. Shen, Z., Andrews, J.G., Evans, B.L.: Optimal Power Allocation in Multiuser OFDM Systems. Proc. IEEE Global Comm. Conference 1 (2003) 337-341

6. Sherali, H.D., Choi, G., Tuncbilek, C.H.: A variable target value method for nondifferentiable optimization. Oper. Res. Lett. 26 (2000) 1-8

7. Wong, C.Y., Cheng, R.S., Letaief, K.B., Murch, R.D.: Multiuser OFDM with adaptive subcarrier, bit, and power allocation. IEEE Journal on Selected Areas in Communications 17 (1999) 1747-1758 